\title{
Differences in gene expression within a striking phenotypic mosaic Eucalyptus tree that varies in susceptibility to herbivory
}

\author{
Amanda Padovan*, Andras Keszei, William J Foley and Carsten Külheim
}

\begin{abstract}
Background: Long-lived trees can accumulate mutations throughout their lifetimes that may influence biotic and abiotic interactions. For example, some Eucalyptus trees display marked variation in herbivore defence within a single canopy. These "mosaic" trees support foliage with distinct chemotypes which are differentially favoured by insect and vertebrate herbivores, resulting in susceptible and resistant branches within a single canopy. These mosaic trees provide a unique opportunity to explore the biosynthesis and genetic regulation of chemical defences in the foliage. The biosynthesis of the principal defence compounds, terpenoid-dominated essential oils, is well understood. However, the regulation of the genes involved and thus the control of phenotypic variation within a single tree canopy remains a mystery.

Results: We sequenced the transcriptomes of the leaves of the two different chemotypes of a chemically mosaic Eucalyptus melliodora tree using 454 pyrosequencing technology. We used gene set enrichment analysis to identify differentially expressed transcripts and found the proportion of differentially expressed genes in the resistant and susceptible foliage similar to the transcript difference between functionally distinct tissues of the same organism, for example roots and leaves. We also investigated sequence differences in the form of single nucleotide polymorphisms and found 10 nucleotides that were different between the two branches. These are likely true SNPs and several occur in regulatory genes.

Conclusion: We found three lines of evidence that suggest changes to a 'master switch' can result in large scale phenotypic changes: 1. We found differential expression of terpene biosynthetic genes between the two chemotypes that could contribute to chemical variation within this plant. 2. We identified many genes that are differentially expressed between the two chemotypes, including some unique genes in each branch. These genes are involved in a variety of processes within the plant and many could contribute to the regulation of secondary metabolism, thus contributing to the chemical variation. 3. We identified 10 SNPs, some of which occur in regulatory genes that could influence secondary metabolism and thus contribute to chemical variation. Whilst this research is inherently limited by sample size, the patterns we describe could be indicative of other plant genetic mosaics.
\end{abstract}

Keywords: Eucalyptus, Mosaic, Somatic mutation, Comparative transcriptomics, Herbivory

\footnotetext{
* Correspondence: amanda.padovan@anu.edu.au

Research School of Biology, Australian National University, Gould Wing, Building No. 116, Canberra ACT 0200, Australia
} 


\section{Background}

Somatic mutations in multicellular organisms can lead to genetically mosaic individuals [1,2]. These mutations occur in a somatic cell line and are due to DNA sequence changes, chromosomal aberrations or epigenetic alterations of DNA [3]. Whereas the genetic changes are interesting, some somatic mutations are truly remarkable because of the striking phenotypic changes that result. Examples include the different coloured flowers of Japanese morning glory (Pharbitis nil) [4] or the disease phenotype associated with cancer in humans [3]. Furthermore, many of our horticultural industries are based on somatic mutations: nectarines are a genetic variant of the peach (Prunus persica) and have co-occurred on a single tree since 1937 [5].

Theoretical models predict that genetic mosaics should comprise just $5 \%$ of a clonal plant population and this rate should be much lower for animals [6]. This is supported by the data on worldwide cancer rates with approximately $0.2 \%$ of the human population having a detected cancer [7]. Therefore, genetic mosaics should be hard to identify, particularly if they are not associated with a phenotypic change or if the phenotypic change is cryptic (e.g. a change in chemical composition of the tissue). Very few genetic mosaics have been described among non-clonal plants and those that have are in crop species, for example peach and nectarine [5]. Striking mosaics have been detected in three Eucalyptus species; E. radiata [8], E. melliodora [9] and E. sideroxylon [10], which all vary in foliar chemical composition. Long lived forest trees, such as Eucalyptus, can accumulate somatic mutations, which may be favourable under certain biotic or abiotic conditions. These mutations may then persist and can influence interactions with other organisms.

Mosaic Eucalyptus trees provide a unique opportunity to investigate specific biosynthetic pathways without the usual challenge of variation between individuals. The transcriptome is one of the best places to look for functional genetic differences because it represents expressed genes and varies with changing conditions [11]. The transcriptomes of different tissues of the same individual are qualitatively and quantitatively different [12], as is the transcriptome of the same tissue from different individuals (of the same species) in similar conditions [13]. Despite this, comparative approaches have succeeded in measuring the response of gene expression to specific changes in the environment, such as drought or salinity stress $[13,14]$. Comparative transcriptomics approaches can employ a variety of technologies to compare and contrast the transcriptomes of two samples, with the aim of identifying pathways or specific genes that differ with the variation in environment [13-15]. This experimental design has become increasingly popular with the advent of next- generation sequencing technologies, and is especially useful for non-model organisms as it does not require a reference genome [16].

We use comparative transcriptomics to investigate differential gene expression, using gene set enrichment analysis (GSEA), between leaves of two chemically different branches of a mosaic Eucalyptus melliodora (yellow box) tree. This tree was identified as a phenotypic mosaic in 1990, when Edwards et al. reported differential defoliation by insect herbivores: insects defoliated most of the tree (ca 95\% defoliation, susceptible chemotype) but left one branch almost untouched (ca 5\% defoliation, resistant chemotype - Figure 1) [9]. Padovan et al. reported consistent and discontinuous differences in three distinct groups of plant secondary metabolites (monoterpenes, sesquiterpenes and formylated phloroglucinol compounds (FPCs)) between the leaves of the two chemotypes [17], which have persisted since the first chemical profiling was done [9]. The chemical profiles of the resistant and susceptible chemotypes differ significantly in these three biosynthetically distinct classes of secondary metabolites, which supports the prediction of Edwards et al. that the chemical patterns observed in the mosaic are due to a somatic mutation in meristematic tissue that was favoured during times of intense herbivory [9].

Here we analyse the transcriptomes of leaves collected from the two chemotypes of the E. melliodora mosaic to investigate the functional genetic differences involved in the contrasting susceptibility to herbivory. The specific aims of this study are to:

1. determine if genes of the terpene and FPC biosynthetic pathways are differentially expressed in leaves of susceptible and resistant branches

2. identify the differentially expressed genes and unique genes in the leaves from each branch

3. identify single nucleotide polymorphisms (SNPs) that could be important in determining the susceptible and resistant phenotypes

\section{Results and Discussion}

\section{Sequencing results and transcriptome assembly}

We used 454 technology to sequence the foliar transcriptome of the two chemotypes on the mosaic $E$. melliodora tree, which yielded 277725 reads (BioSample Project BSH193). Attempts to assemble these reads to the Eucalyptus grandis genome sequence with either CLC Genomics Workbench (53\% of reads assembled) or Lastz (60\% of reads assembled; in Galaxy [18]) were unsatisfactory. Thus we used the de novo assembly option in CLC Genomics Workbench to assemble $88 \%$ of reads into 13072 contigs with an average length of 616 bp (Table 1, Additional file 1: Figure S1). Our limited success in assembling the reads against the E. grandis 


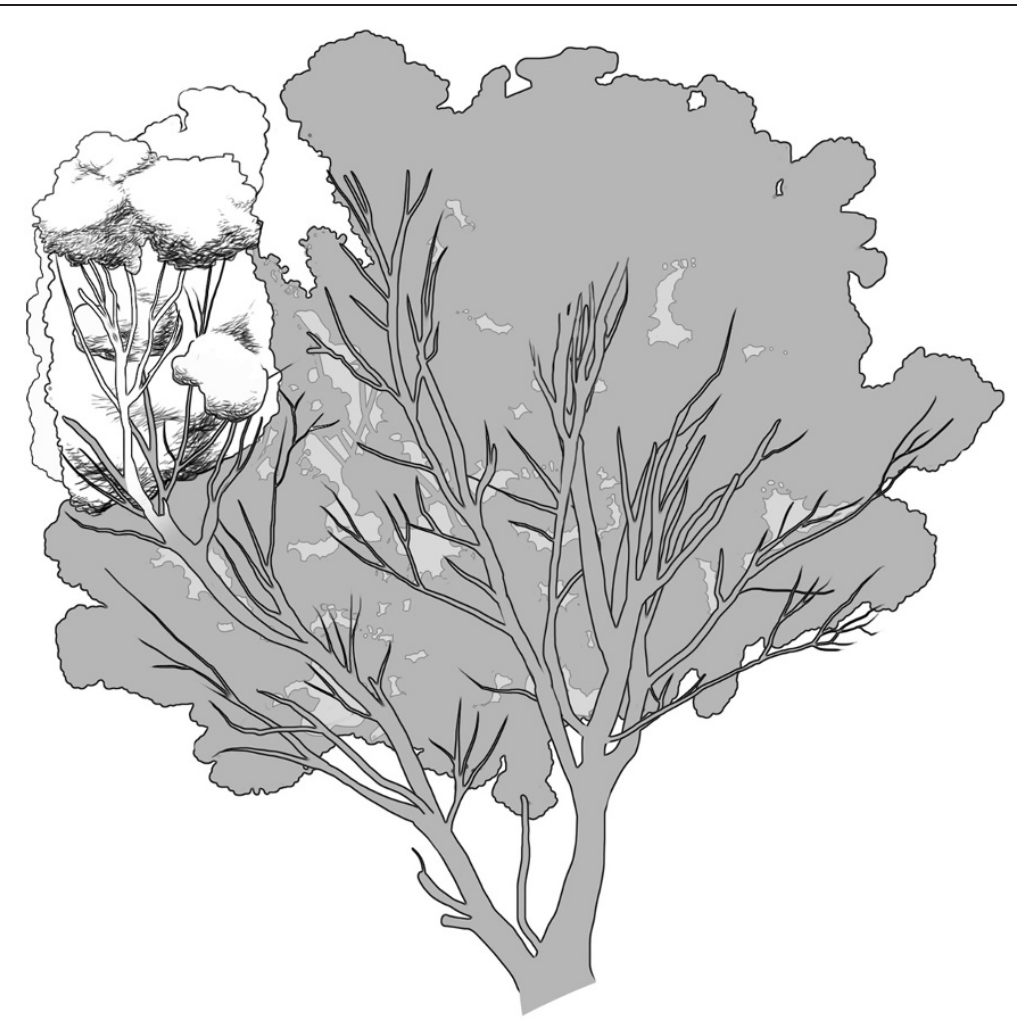

Figure 1 The mosaic Eucalyptus melliodora tree after an insect outbreak (Walsh E, 2012). The grey area represents foliage susceptible to herbivory, and the white area represents foliage that is resistant to herbivory. Each area has an associated chemical profile [9,17].

genome is probably due to the long reads generated using 454 technology. The software (Lastz and CLC genomics workbench) was optimised to map short reads against a genome and was unable to successfully map the longer reads generated using this method of sequencing.

Table 1 Sequencing statistics generated by de novo assembly for the reads generated by sequencing the mRNA pool of leaves from the resistant and susceptible branches of a mosaic Eucalyptus melliodora

\begin{tabular}{|c|c|c|c|c|}
\hline & & Counts & Mean length & Total bases \\
\hline \multirow{4}{*}{$\begin{array}{l}\text { de novo } \\
\text { assembly (i) }\end{array}$} & total reads & 277725 & 296 & 82179685 \\
\hline & mapped reads & 243470 & 290 & 70654843 \\
\hline & unmapped reads & 34255 & 336 & 11524842 \\
\hline & contigs & 13104 & 599 & 7853262 \\
\hline \multirow{4}{*}{$\begin{array}{l}\mathrm{R} \text { mapped to } \\
\text { de novo (ii) }\end{array}$} & total reads & 133574 & 320 & 42788130 \\
\hline & mapped reads & 114093 & 316 & 36061899 \\
\hline & unmapped reads & 19481 & 345 & 6726231 \\
\hline & contigs & 11965 & 615 & 7367368 \\
\hline \multirow{4}{*}{$\begin{array}{l}\text { S mapped to } \\
\text { de novo (iii) }\end{array}$} & total reads & 144151 & 273 & 39391555 \\
\hline & mapped reads & 130008 & 267 & 34767476 \\
\hline & unmapped reads & 14143 & 327 & 4624079 \\
\hline & contigs & 10130 & 626 & 6350291 \\
\hline
\end{tabular}

(i) contains all reads from this library, (ii) contains all reads over-represented in the resistant branch compared with the susceptible branch and (iii) contains all reads over-represented in the susceptible branch compared with the resistant branch.

\section{Identifying the transcripts through BLAST and GO classification}

We used Blast2GO [19] to identify the best BLAST hit and to determine the gene ontology (GO) classification for each transcript. There were no BLAST hits for 30\% of the contigs (3841 contigs) and no annotation or GO categories could be determined for $9 \%$ of the contigs (1199 contigs).

We divided our data into three sets: (i) the reference set which contains all the transcripts sequenced from leaves of the two chemotypes, (ii) all the transcripts over-represented in the leaves of the resistant branch compared with the leaves of the susceptible branch (overR), and (iii) all the transcripts over-represented in the leaves of the susceptible branch compared with the leaves of the resistant branch (overS). Datasets (ii) and (iii) were compiled from fold-change data generated using the expression analysis menu of CLC Genomics Workbench with a minimum threshold of a 2.5 fold difference in expression of the transcripts. For all three datasets most BLAST hits were from Vitis vinifera followed by Populus trichocarpa and Arabidopsis thaliana, (data not shown). Although Eucalyptus and Populus are more related to each other than to Vitis [20] and there are more genes from Populus (44 852) than from Vitis at NCBI (36 934) - Gene database, National Center 
Table 2 Summary of the within tree foliar chemical variation (adapted from Table 2 by Padovan et al. [17])

\begin{tabular}{|c|c|c|c|c|}
\hline & \multicolumn{2}{|r|}{ Resistant } & \multicolumn{2}{|r|}{ Susceptible } \\
\hline & Concentration & $\begin{array}{r}\text { No. of } \\
\text { Compounds }\end{array}$ & Concentration. & $\begin{array}{r}\text { No. of } \\
\text { Compounds }\end{array}$ \\
\hline Monoterpenes & $12.2(1.18)$ & 10 & $6.3(1.55)$ & 15 \\
\hline Sesquiterpenes & $1.7(1.3)$ & 7 & $24.2(4.6)$ & $\overline{16}$ \\
\hline$\overline{\mathrm{FPCS}}$ & $5.4(0.3)$ & 3 & $0.26(0.3)$ & 3 \\
\hline
\end{tabular}

From each branch (resistant and susceptible) we have reported the concentration and standard deviation across five branches (in $\mathrm{mg} \cdot \mathrm{g}^{-1} \mathrm{dry}$ weight) and number of compounds in the three biosynthetically distinct classes of defence chemicals: monoterpenes, sesquiterpenes and formylated phloroglucinol compounds (FPCs).

for Biotechnology Information, U.S. National Library of Medicine), Vitis still provided more BLAST hits than did Populus. Vitis is rich in secondary metabolites and supports the greatest diversity of terpene synthase genes after Eucalyptus grandis [21], which may explain this result. An inherent limitation of this paper is the level of replication; there is no scope for independent replication experiments since the variation occurs within a single tree. Whilst this is a limitation, the work we are doing with this tree contributes significantly to our understanding of the evolution of variation in plant secondary metabolites.

We used the three datasets to address our aims.

\section{Are terpene and FPC biosynthetic genes differentially expressed in leaves of the resistant and susceptible chemotypes?}

The resistant and susceptible branches of the mosaic tree have a distinct foliar chemical profile with major differences in terpenes and FPCs [17]. Leaves of the resistant chemotype have a higher concentration of monoterpenes and FPCs, while leaves of the susceptible chemotype have a higher concentration of sesquiterpenes (Table 2) [17]. Therefore, we expected to find genes involved in the methyl erythritol pyrophosphate (MEP) pathway and FPC biosynthesis, as well as monoterpene synthases that are significantly up-regulated in the leaves of the resistant chemotype compared with those of the susceptible chemotype. In contrast, genes involved in the mevalonate (MVA) pathway and sesquiterpene synthases should be significantly up-regulated in the leaves of the susceptible chemotype compared with those of the resistant chemotype (for a detailed summary of genes involved in terpene and FPC biosynthesis see Külheim et al. [22]). We identified contigs that aligned to genes of interest and compared the relative expression levels in each library. As predicted, in the transcriptome of the resistant leaves there was an over-abundance of transcripts associated with the MEP pathway, as well as an over-abundance of terpene synthases and FPC biosynthetic transcripts, but some individual transcripts were more abundant in the transcriptome of the susceptible leaves.

We identified 26 different transcript sequences for putative terpene synthase transcripts, using the best BLAST hit, in the entire library of 13,072 contigs: seven monoterpene synthases, 15 sesquiterpene synthases and three triterpene synthases. These transcripts are rare, which makes statistical analyses problematic. However, there are up to 10 putative terpene synthase transcripts that are differentially expressed between the two branches: two triterpene synthases, three monoterpene

Table 3 Statistical analysis (Fisher's exact test) of the over or under representation of GO categories in the overR (ii) data set derived from the transcriptome of leaves from resistant branches of a mosaic Eucalyptus melliodora tree

\begin{tabular}{|c|c|c|c|c|c|}
\hline GO Term & Name & Type & FDR & p-Value & Over/Under \\
\hline GO:0003824 & catalytic activity & $\mathrm{F}$ & 0.45 & 0.00 & + \\
\hline GO:0007049 & cell cycle & $P$ & 0.56 & 0.02 & + \\
\hline GO:0005198 & structural molecule activity & $\mathrm{F}$ & 0.56 & 0.02 & - \\
\hline GO:0005840 & ribosome & C & 0.56 & 0.04 & - \\
\hline GO:0030529 & ribonucleoprotein complex & C & 0.56 & 0.04 & - \\
\hline GO:0000166 & nucleotide binding & $F$ & 0.56 & 0.04 & - \\
\hline GO:0005216 & ion channel activity & $F$ & 0.56 & 0.04 & + \\
\hline GO:0015075 & ion transmembrane transporter activity & $\mathrm{F}$ & 0.56 & 0.04 & + \\
\hline GO:0015267 & channel activity & $\mathrm{F}$ & 0.56 & 0.04 & + \\
\hline GO:0022803 & passive transmembrane transporter activity & $\mathrm{F}$ & 0.56 & 0.04 & + \\
\hline GO:0022838 & substrate-specific channel activity & $\mathrm{F}$ & 0.56 & 0.04 & + \\
\hline GO:0022857 & transmembrane transporter activity & $\mathrm{F}$ & 0.56 & 0.04 & + \\
\hline GO:0022891 & substrate-specific transmembrane transporter activity & $\mathrm{F}$ & 0.56 & 0.04 & + \\
\hline GO:0022892 & substrate-specific transporter activity & $\mathrm{F}$ & 0.56 & 0.04 & + \\
\hline GO:0005975 & carbohydrate metabolic process & $P$ & 0.56 & 0.04 & + \\
\hline
\end{tabular}

Type refers to the GO category type: $\mathrm{F}=$ molecular function, $\mathrm{P}=$ biological processes and $\mathrm{C}=$ cellular component. FDR refers to the false discovery rate (FDR)corrected p-value. $\mathrm{p}$-Value refers to single test $\mathrm{p}$-value. In the over/under column, + signifies over-represented categories and - signifies under-represented categories when compared to the reference data set (i). 
Table 4 Statistical analysis (Fisher's exact test) of the over or under representation of GO categories in the overS (iii) data set derived from the transcriptome of leaves from susceptible branches of a mosaic Eucalyptus melliodora tree

\begin{tabular}{|c|c|c|c|c|c|}
\hline GO Term & Name & Type & FDR & p-Value & Over/Under \\
\hline GO:0010468 & regulation of gene expression & $P$ & 0.01 & 0.00 & + \\
\hline GO:0019222 & regulation of metabolic process & $P$ & 0.01 & 0.00 & + \\
\hline GO:0040029 & regulation of gene expression, epigenetic & $\mathrm{P}$ & 0.01 & 0.00 & + \\
\hline GO:0060255 & regulation of macromolecule metabolic process & $P$ & 0.01 & 0.00 & + \\
\hline GO:0005840 & ribosome & C & 0.01 & 0.00 & - \\
\hline GO:0030529 & ribonucleoprotein complex & $\mathrm{C}$ & 0.01 & 0.00 & - \\
\hline GO:0005198 & structural molecule activity & $\mathrm{F}$ & 0.03 & 0.00 & - \\
\hline GO:0005488 & binding & $\mathrm{F}$ & 0.03 & 0.00 & + \\
\hline GO:0006139 & nucleobase, nucleoside, nucleotide and nucleic acid metabolic process & $P$ & 0.04 & 0.00 & + \\
\hline GO:0006807 & nitrogen compound metabolic process & $\mathrm{P}$ & 0.04 & 0.00 & + \\
\hline GO:0034641 & cellular nitrogen compound metabolic process & $\mathrm{P}$ & 0.04 & 0.00 & + \\
\hline GO:0009719 & response to endogenous stimulus & $\mathrm{P}$ & 0.06 & 0.00 & + \\
\hline GO:0000166 & nucleotide binding & $\mathrm{F}$ & 0.07 & 0.00 & + \\
\hline GO:0043226 & organelle & $\mathrm{C}$ & 0.07 & 0.00 & - \\
\hline GO:0005622 & intracellular & C & 0.09 & 0.01 & - \\
\hline GO:0043229 & intracellular organelle & C & 0.09 & 0.01 & - \\
\hline GO:0044444 & cytoplasmic part & C & 0.10 & 0.01 & - \\
\hline GO:0006412 & translation & $P$ & 0.10 & 0.01 & - \\
\hline GO:0009059 & macromolecule biosynthetic process & $P$ & 0.10 & 0.01 & - \\
\hline GO:0034645 & cellular macromolecule biosynthetic process & $P$ & 0.10 & 0.01 & - \\
\hline GO:0044249 & cellular biosynthetic process & $P$ & 0.10 & 0.01 & - \\
\hline GO:0044424 & intracellular part & $\mathrm{C}$ & 0.12 & 0.01 & - \\
\hline GO:0005737 & cytoplasm & C & 0.13 & 0.01 & - \\
\hline GO:0044464 & cell part & C & 0.13 & 0.01 & - \\
\hline GO:0043412 & macromolecule modification & $\mathrm{P}$ & 0.15 & 0.02 & + \\
\hline GO:0016788 & hydrolase activity, acting on ester bonds & $\mathrm{F}$ & 0.15 & 0.02 & + \\
\hline GO:0016301 & kinase activity & $\mathrm{F}$ & 0.15 & 0.03 & + \\
\hline GO:0016772 & transferase activity, transferring phosphorus-containing groups & $\mathrm{F}$ & 0.15 & 0.03 & + \\
\hline GO:0003774 & motor activity & $\mathrm{F}$ & 0.15 & 0.03 & + \\
\hline GO:0016462 & pyrophosphatase activity & $\mathrm{F}$ & 0.15 & 0.03 & + \\
\hline GO:0016817 & hydrolase activity, acting on acid anhydrides & $\mathrm{F}$ & 0.15 & 0.03 & + \\
\hline GO:0016818 & hydrolase activity, acting on acid anhydrides, & $\mathrm{F}$ & 0.15 & 0.03 & + \\
\hline GO:0017111 & nucleoside-triphosphatase activity & $\mathrm{F}$ & 0.15 & 0.03 & + \\
\hline GO:0032501 & multicellular organismal process & $P$ & 0.15 & 0.03 & + \\
\hline GO:0043227 & membrane-bounded organelle & C & 0.15 & 0.02 & - \\
\hline GO:0043231 & intracellular membrane-bounded organelle & C & 0.15 & 0.02 & - \\
\hline GO:0043228 & non-membrane-bounded organelle & C & 0.15 & 0.03 & - \\
\hline GO:0043232 & intracellular non-membrane-bounded organelle & $\mathrm{C}$ & 0.15 & 0.03 & - \\
\hline GO:0006464 & protein modification process & $P$ & 0.19 & 0.03 & + \\
\hline GO:0007275 & multicellular organismal development & $\mathrm{P}$ & 0.20 & 0.04 & + \\
\hline GO:0004721 & phosphoprotein phosphatase activity & $\mathrm{F}$ & 0.20 & 0.04 & + \\
\hline GO:0016791 & phosphatase activity & $\mathrm{F}$ & 0.20 & 0.04 & + \\
\hline GO:0042578 & phosphoric ester hydrolase activity & $\mathrm{F}$ & 0.20 & 0.04 & + \\
\hline GO:0032991 & macromolecular complex & C & 0.20 & 0.04 & - \\
\hline GO:0050896 & response to stimulus & $P$ & 0.21 & 0.04 & + \\
\hline GO:0032502 & developmental process & $\mathrm{P}$ & 0.21 & 0.05 & + \\
\hline GO:0009058 & biosynthetic process & $P$ & 0.21 & 0.04 & - \\
\hline
\end{tabular}

Type refers to the $\mathrm{GO}$ category type: $\mathrm{F}=$ molecular function, $\mathrm{P}=$ biological processes and $\mathrm{C}=$ cellular component. FDR refers to the false discovery rate (FDR)corrected $p$-value and the thick horizontal line shows a significance level of 0.05 FDR. p-Value refers to single test $p$-value. In the over/under column, + signifies over-represented categories and - signifies under-represented categories when compared to the reference data set (i). 
synthases and five sesquiterpene synthases. Just two terpene synthase transcripts are more abundant in the leaves of the susceptible branch than in the resistant branch: a mono- and a sesquiterpene synthase. This is surprising given that susceptible leaves had a higher concentration of sesquiterpenes and a greater overall diversity of mono- and sesquiterpenes (Table 2).

There are seven genes involved in the MEP pathway, which provides the precursors for monoterpene synthesis [23]. We identified at least two contigs for six of the genes in the MEP pathway. There are no contigs matching $m c s$, the fifth step in the pathway. Four transcripts are more abundant in the transcriptome of the resistant leaves than the susceptible leaves (one copy each of $d x r, m c t, c m k$, $h d r)$. Transcripts of these genes correlate strongly with monoterpene production in a closely related tree (Melaleuca alternifolia) [24] which is consistent with the higher concentration of monoterpenes in the leaves of the resistant chemotype than leaves of the susceptible chemotype (Table 2).

There are four genes involved in the MVA pathway, which provides the precursors for sesquiterpene synthesis [23] and we identified contigs that align to the final two genes $(m v k$ and $p m d)$. All of these contigs were equally prevalent in the transcriptomes from the resistant and susceptible leaves. This is surprising since leaves of the susceptible chemotype contain higher concentrations of sesquiterpenes than those of the resistant chemotype, with the opposite pattern found for monoterpenes (Table 2). Several studies have shown that the precursors, IPP and DMAPP, produced by the MEP pathway can be transported across the plastidal membrane and used in sesquiterpene production [24,25]. This could explain the chemical patterns shown for this tree (Table 2).

\section{What other genes are differentially expressed between leaves of the resistant and susceptible chemotypes?}

The two libraries from the resistant (overR - ii) and susceptible (overS - iii) chemotypes were mapped against the reference sequence of the combined libraries (i). Gene expression in the same tissue taken from the same individual usually shows little or no difference between samples. For example the transcriptome of sperm cells taken from an Arabidopsis thaliana plant share 97\% identity [26]. Even studies that compare individuals of the same chemotype tend to find few differences [27]: single gene null function mutants in Arabidopsis showed little difference in global gene expression [28]. Therefore, we were surprised to find a large proportion of genes differentially expressed between the two chemotypes. We found 436 transcripts (3.3\%) that were over-expressed at least 10-fold in the resistant branch, while 970 transcripts $(7.4 \%)$ were similarly over-expressed in the susceptible branch (Table 2). This difference compares to the variation in gene expression found between functionally distinct tissues in the same organism [29].

We further investigated specific transcripts that were differentially expressed in the transcriptomes of the two chemotypes. A 2.5 fold threshold and a 10-fold threshold identified the same transcripts as being over and underrepresented in the two chemotypes, suggesting that a major reorganisation of gene expression had occurred. The volcano plot (Additional file 2: Figure S2) shows that many genes were significantly differentially expressed between leaves from the resistant and susceptible chemotypes. We used fold-change data, generated in CLC Genomics Workbench, to compile lists of contigs overexpressed in leaves from the resistant and susceptible chemotype. There were many more genes over-expressed in leaves from the susceptible branch (970) compared with

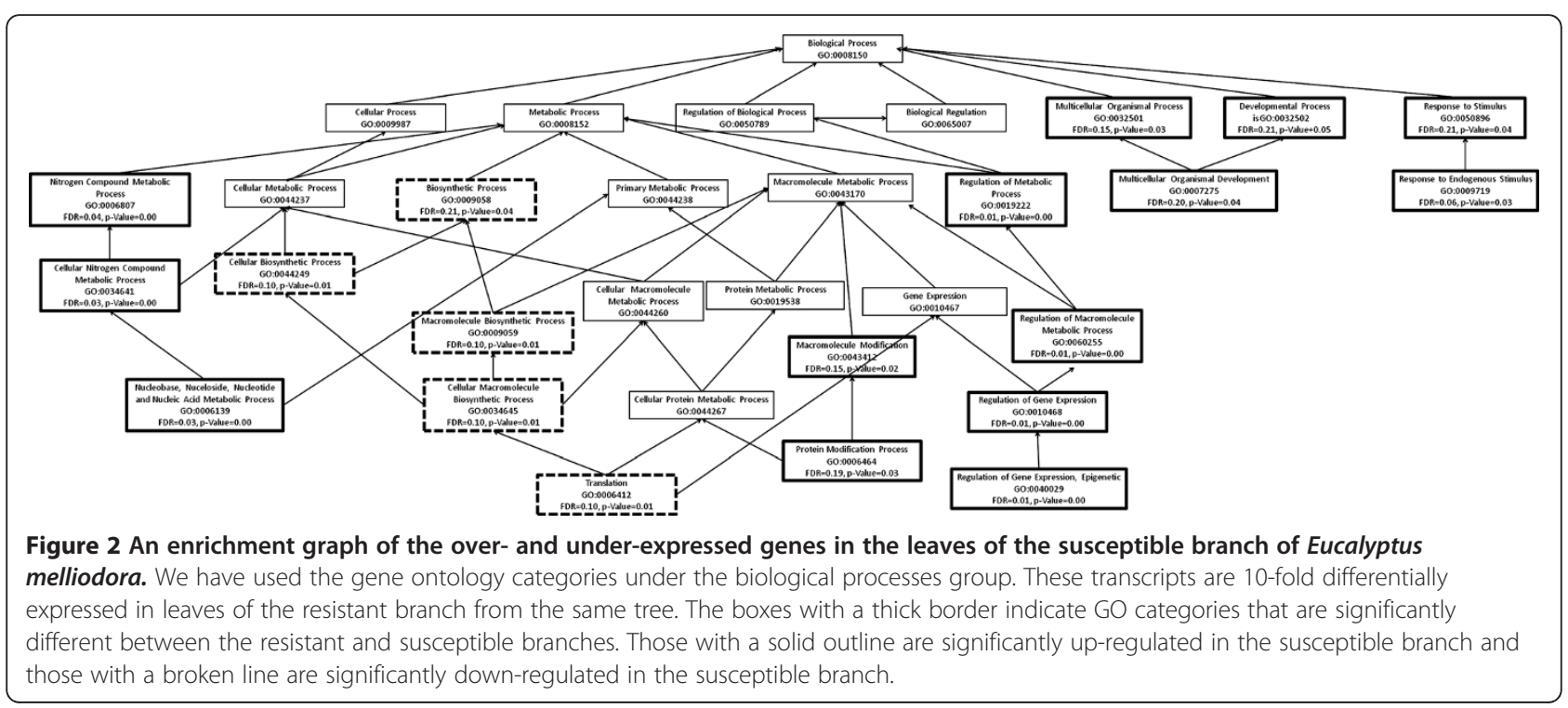


leaves from the resistant branch (436). We used Fisher's Exact Test to determine whether the GO categories of expressed genes were significantly enriched in leaves from the two chemotypes. We used a FDR (false discovery rate)-corrected $P$-value of 0.05 to determine significance of differential expression (Table 3 and Table 4) and the standard $P$-value of significance to show overall patterns of differential gene expression (Figures 2, 3, 4, 5).

We observed an up-regulation of transcripts with trans-membrane transport and channel activity in the leaves of the resistant chemotype (Figures 2 and 3). Transcripts with nucleotide binding, structural molecular activity and those associated with ribosomes and their complexes were down-regulated in these leaves (Figures 2 and 3). This suggests that metabolites are being transported across membranes [30] and that there is less interaction between nucleic acids and regulators, modifiers and replication machinery resulting in a decrease in overall gene expression (Table 3 ).

The genes up-regulated in the leaves of the susceptible chemotype, include those involved in the regulation of gene expression and metabolism, macromolecule modification and response to endogenous stimulus (FDRcorrected $P$-value, see Table 4). These include genes with specific transferase, kinase, phosphatase and hydrolase activity (Figures 4 and 5). Genes involved in translation

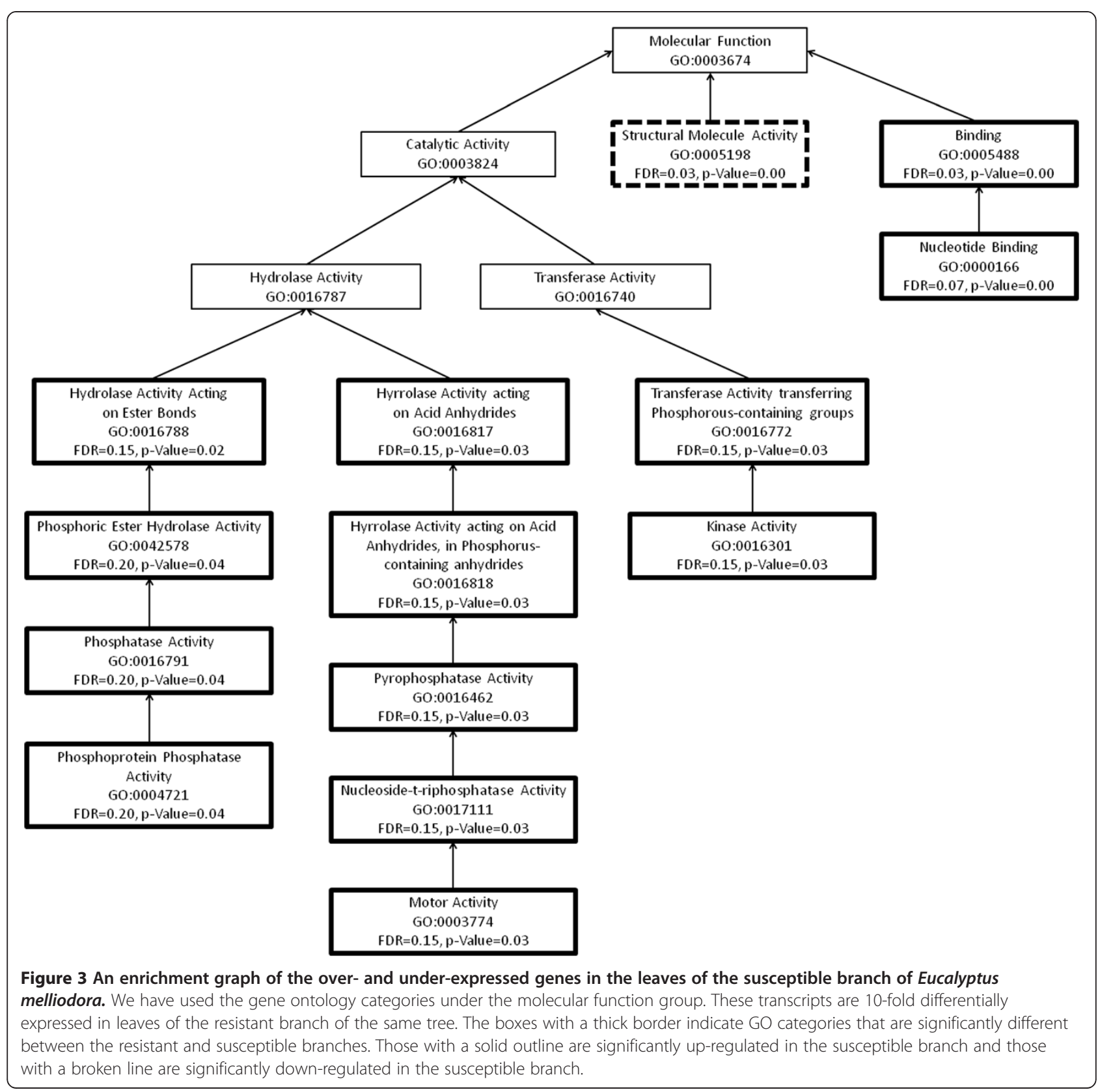




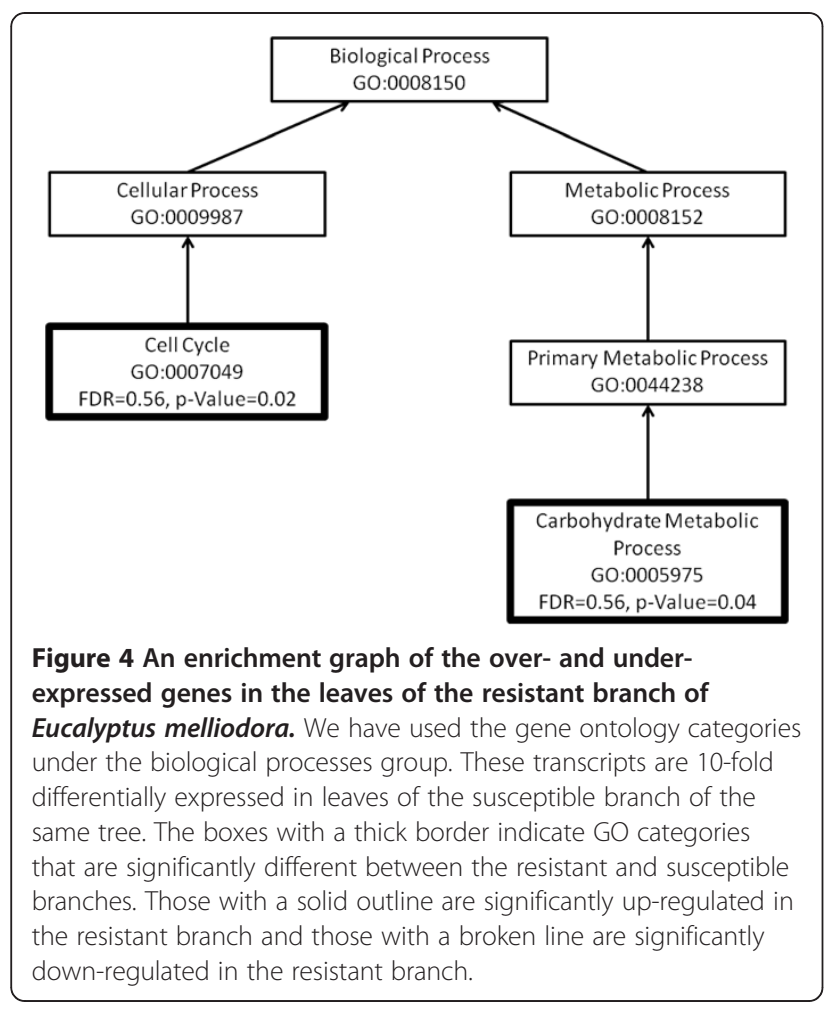

and macromolecular biosynthesis were down-regulated in the leaves of the susceptible chemotype (Figures 4 and 5). This suggests there is a tightly controlled mechanism responding to stimuli that involves changes to gene expression and protein modification in leaves of the susceptible branch. This is expected due to the complexity of the sesquiterpene profile of the susceptible leaves: the oil from these leaves contains 16 sesquiterpenes, compared with seven sesquiterpenes in the foliar oil of the resistant chemotype. Each of these compounds is associated with a gene which must be regulated [31].

We found 455 contigs that are unique to the transcriptome of leaves of the susceptible chemotype and 1645 contigs that are unique to the transcriptome of leaves of the resistant chemotype. The transcriptome of the resistant leaves contain more unique genes, excluding regulatory genes, than does that of the susceptible leaves and since these genes require control, there are also many more regulatory genes in the transcriptome of the resistant chemotype (54:5). The leaves of the resistant chemotype also contain a much higher concentration of FPCs than the leaves of the susceptible chemotype which is represented by the number of unique FPC biosynthesis genes in the transcriptome of the resistant chemotype (8:0). The number of unique terpene biosynthetic genes in the transcriptome of the resistant branch is somewhat surprising given that the leaves of the susceptible chemotype have twice the number of terpenes and the terpene concentration was much higher than the leaves of the resistant chemotype (7:1 - Table 2).

\section{Identifying single nucleotide polymorphisms (SNPs) that could play a role in the two chemotypes}

We found 10 putative single nucleotide polymorphisms (SNPs) between the leaves of the resistant chemotype compared with the leaves of the susceptible chemotype (using CLC Genomics Workbench - Table 5). This is much smaller than the number of SNPs that differ between species: for example, the closely related $E$. globulus and E. nitens have 1478 and 1418 SNPs in genes of the secondary metabolism pathway [22] respectively, a difference of 60 SNPs. The proportion of transitions and transversions in these 10 loci (Figure 6) matches the proportions for SNPs in the entire Arabidopsis genome [32], making it likely that these are true SNPs. Different mutations occur at different frequencies and the observed frequencies (E. melliodora) match the expectations (Arabidopsis thaliana). Although the data set is small, it is nevertheless encouraging.

SNPs in two genes, identified by their best BLAST hit, could be involved in epigenetic regulation: methyl cpg binding domain 10 and histone deacetylase complex subunit sap18. Both of these transcripts are more abundant in leaves from the susceptible chemotype than the resistant chemotype and they are homozygous in leaves of the resistant branch, but heterozygous in leaves of the susceptible chemotype. This may be the result of differential allelic expression of certain genes in the resistant leaves compared with the susceptible leaves. In humans, mice and maize, differential allelic expression has been implicated in quantitative trait variation and gene regulation which may result in phenotypic variation [33,34]. These SNPs indicate a role for epigenetic regulation in the formation and maintenance of the mosaic eucalypt and that is an area for future research.

\section{Are these results representative of other phenotypic mosaics?}

Whilst there are only a few examples of phenotypic mosaicism in plants, and our focus has been on Eucalyptus, we expect there are many more phenotypic mosaics in natural populations that are yet to be identified. In this report we have focussed on the differences in terpene biosynthetic genes and possible regulatory genes that could explain the chemical variation described [17] and the patterns we have described are likely indicative of gene expression in other plants with similarly high terpene concentrations and variability. However, the idea that the two parts of a mosaic organism have very different gene expression patterns, particularly in those pathways involved in the phenotype, is likely to apply to all phenotypic mosaics.

\section{Conclusions}

Long-lived modular organisms can accumulate somatic mutations over their lifetime enabling them to adjust to a changing environment. Small changes to a 'master switch' 


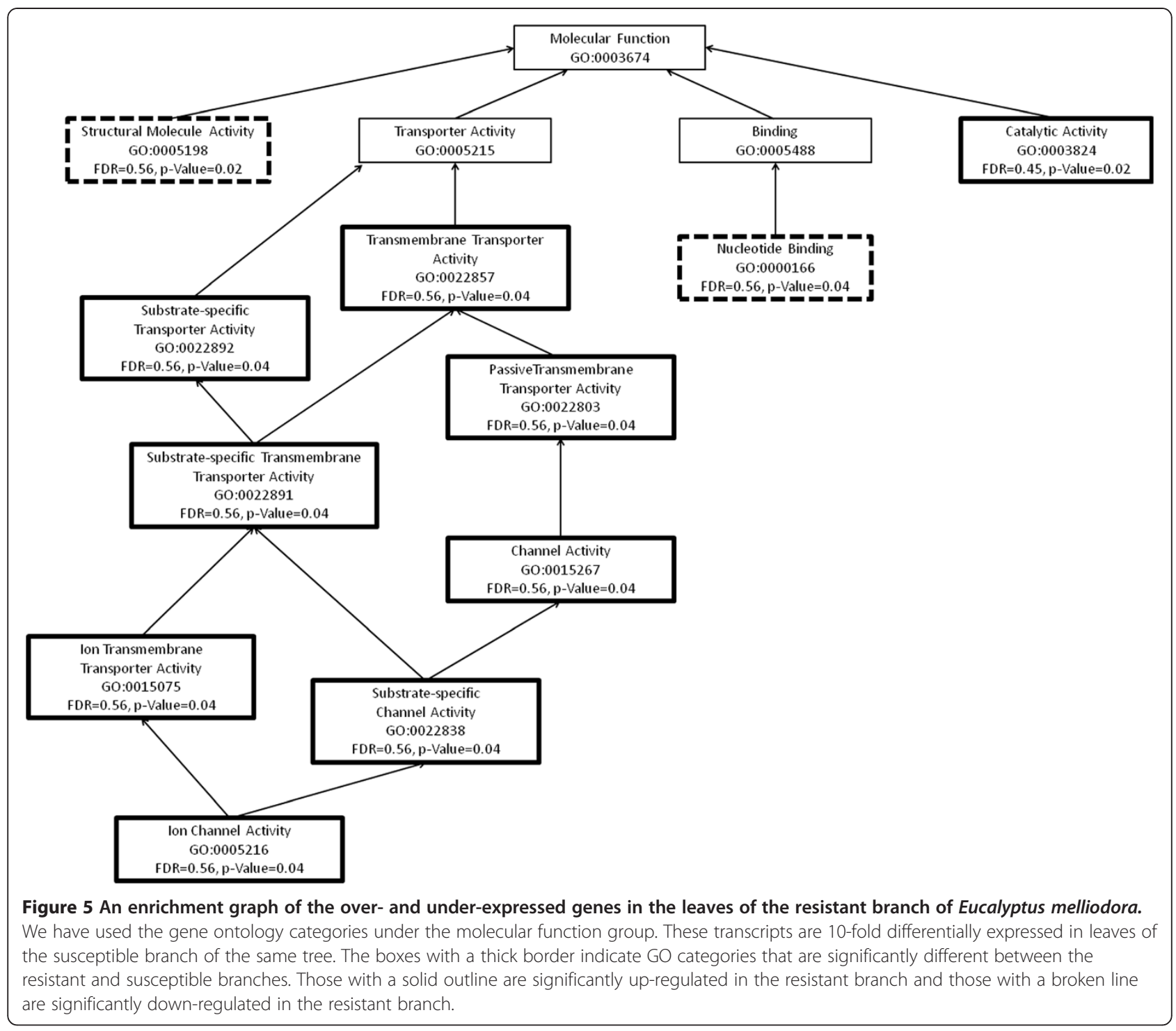

Table 5 The most abundant SNPs with differential representation in the transcriptomes of the resistant (R) and susceptible (S) branches of Eucalyptus melliodora

\begin{tabular}{|c|c|c|c|}
\hline Contig - position & $\mathrm{R}$ allele & S allele & BLAST Hit \\
\hline contig $12190-233$ & G/G (28/-) & $\mathrm{G} / \mathrm{A}(25 / 6)$ & methyl cpg binding domain 10 \\
\hline contig $5065-502$ & $\mathrm{~T} / \mathrm{C}(18 / 16)$ & $\mathrm{T} / \mathrm{T}(2 /-)$ & peptidase $\mathrm{m} 1$ family protein \\
\hline contig $5021-317$ & G/G (10/-) & G/A (17/4) & histone deacetylase complex subunit sap 18 \\
\hline contig $5063-668$ & $\mathrm{~T} / \mathrm{G}(11 / 8)$ & $\mathrm{T} / \mathrm{T}(11 /-)$ & pyruvate kinase \\
\hline contig $7410-589$ & $\mathrm{G} / \mathrm{A}(15 / 3)$ & $\mathrm{G} / \mathrm{G}(12 /-)$ & ycf20-like protein \\
\hline contig $11898-31$ & A/G (15/3) & A/A (12/-) & protein \\
\hline contig $12260-395$ & $\mathrm{C} / \mathrm{A}(13 / 2)$ & C/C (6/-) & - \\
\hline contig $4834-463$ & $\mathrm{C} / \mathrm{C}(3 /-)$ & $\mathrm{C} / \mathrm{T}(13 / 2)$ & - \\
\hline contig $7446-648$ & $\mathrm{G} / \mathrm{A}(9 / 7)$ & G/G (2/-) & protein transport protein sec 13 \\
\hline contig $7510-46$ & $\mathrm{G} / \mathrm{G}(4 /-)$ & $\mathrm{G} / \mathrm{A}(11 / 3)$ & - \\
\hline
\end{tabular}

Contig - position shows the contig number and the nucleotide position that is variable. In both the $R$ allele and $S$ allele columns, the variable nucleotides are written first followed by the number of reads at that position for each variant respectively. BLAST Hit shows the best BLAST Hit for each contig (found using Blast2GO [19]) and - represents contigs that had no best BLAST Hit. 


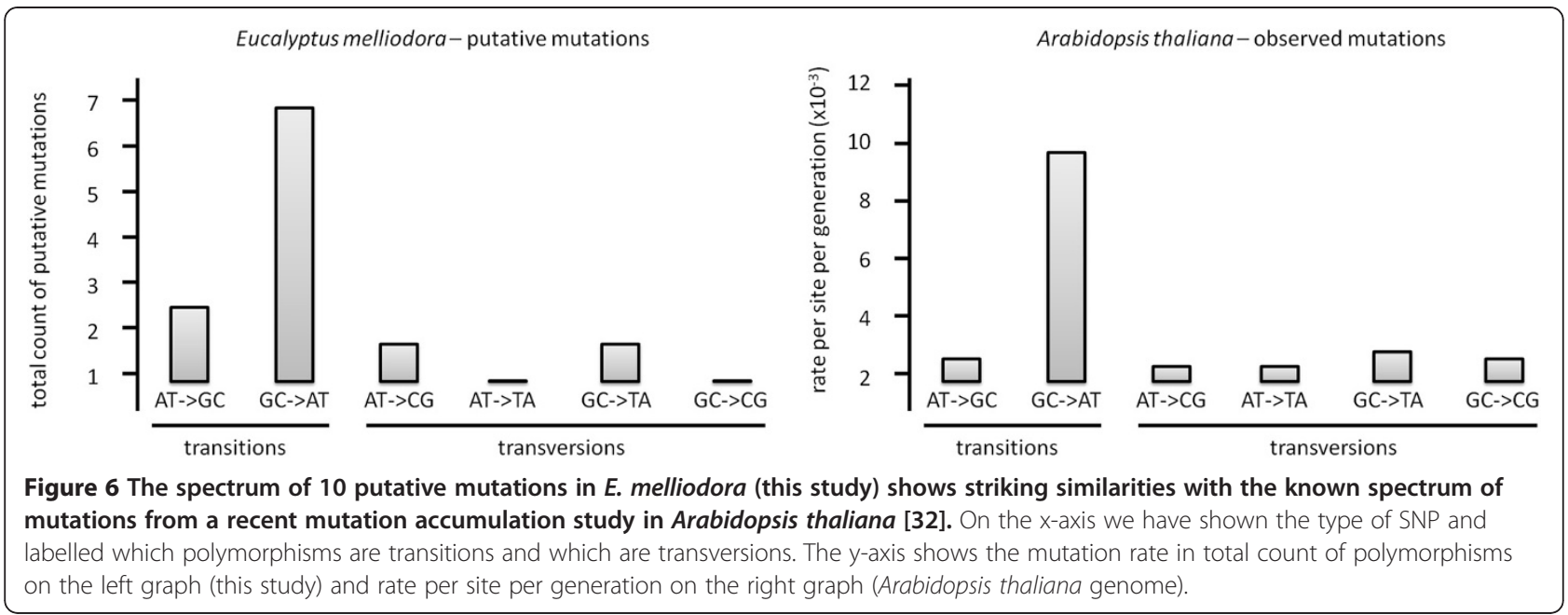

can lead to large-scale changes to gene expression resulting in the same tissue on the same organism becoming functionally distinct. We found three lines of evidence for this in a mosaic Eucalyptus melliodora tree that experiences differential herbivory as a result of chemical diversity: 1 . We found differential expression of terpene biosynthetic genes between the two chemotypes that could contribute to chemical variation within this plant. 2. We identified many genes that are differentially expressed between the two chemotypes, including some unique genes in each branch. These genes are involved in a variety of processes within the plant and many could contribute to the regulation of secondary metabolism and thus contribute to the chemical variation between the two chemotypes. 3 . We identified 10 SNPs that are likely true SNPs and not artefacts of data collection and analysis. Some of these SNPs occur in regulatory genes that could influence secondary metabolism and thus contribute to foliar terpene variation in this plant. Whilst this research is inherently limited by sample size, the patterns we describe could be indicative of other plant genetic mosaics.

\section{Methods}

\section{Plant material}

Foliage samples were collected at a site at Yeoval (NSW, Australia: $\left.32^{\circ} 45^{\prime} 00.00^{\prime \prime} \mathrm{S} 148^{\circ} 39^{\prime} 00.00^{\prime \prime} \mathrm{E}\right)$, known to contain a previously identified as a chemical mosaic Eucalyptus melliodora $[9,10]$. Using a truck-mounted hydraulic-lifted platform, we collected samples of the leaves representing the resistant and susceptible chemotypes of the mosaic. The leaves were immediately put into labelled paper envelopes before being snap frozen in liquid nitrogen. They were subsequently stored at $-80^{\circ} \mathrm{C}$.

\section{RNA extraction}

The leaves were first ground to a fine powder in liquid nitrogen using a mortar and pestle. We used the QIAgen
Plant RNeasy kit to extract RNA (QIAGEN, Valencia California). We followed the manufacturer's instructions, but added $50 \mu \mathrm{l}$ of $20 \%$ polyvinylpyrrolidone (PVP) and $108 \mathrm{mg}$ of sodium isoascorbate (Na-iASC) to the lysis buffer to remove phenolic compounds and polysaccharides that interfere with RNA extraction and downstream applications [35-37]. We used the Oligotex Direct mRNA Mini Kit (Qiagen, Valencia California) to purify mRNA from these samples, following the manufacturer's instructions.

\section{cDNA library synthesis}

We used the SMARTer RACE cDNA Amplification Kit (Clontech, Mountain View California) to generate a cDNA library following manufacturer's instructions. We generated one library from leaves of the resistant branch (the resistant library) and one library from leaves of the susceptible branch (the susceptible library). These libraries were not normalised because this may introduce errors in estimating allele frequency and gene expression. Also, low abundance transcripts (like terpene biosynthetic genes) are likely to be lost [38].

\section{Roche GS FLX Sequencing}

cDNA libraries were nebulised and sequenced on a Roche Applied Sciences GS-FLX according to standard procedure (Roche, Indianapolis, IN). The reads were base-called using 454 software, imported into CLC Genomics Workbench (CLC Bio, Denmark) and truncated to remove low quality bases.

\section{Data Analysis}

First, we assembled the library in CLC Genomics Workbench and then assembled the library using Galaxy (Lastz, [18]). In both cases we used the E. grandis genome as a reference. With the previous methods leading to limited success (percentage of reads aligned to the 
reference genome were below 50\%), we then used CLC Genomics Workbench to do a de novo assembly of the reads and Blast2GO [19] to identify the best BLAST hit and to determine the gene ontology (GO) classification for each transcript, using the default settings. We did an enrichment analysis of the over abundant contigs from both the resistant and susceptible libraries against the total library using the Fishers Exact Test (false discovery rate-corrected $P$-value) and generated enrichment plots ( $P$-value) of these data in Blast2Go [19].

\section{SNP discovery}

Using CLC Genomics Workbench (CLC Bio, Denmark), we first identified heterozygous sites within the combined de-novo assembly with a minimum of three reads from the minor allele. Then, both assemblies for R and S cDNA libraries were searched at the loci which were identified as heterozygous and identified as heterozygous (same as combined) or homozygous for either allele.

\section{Additional files}

Additional file 1: Figure S1. A histogram of the length of 13,072 contigs generated from the library of transcripts from Eucalyptus melliodora leaves of different chemotypes. The average length is $616 \mathrm{bp}$.

Additional file 2: Figure S2. Volcano plot of transcripts from the leaves of resistant branch of Eucalyptus melliodora against the transcripts from the leaves of the susceptible branch of the same tree. The $x$-axis shows fold change, with positive values representing transcripts over-expressed in the leaves of the resistant branch. The $y$-axis represents the significance level with a larger value indicating a higher significance. A value of 5 on the $y$-axis represents a p-value of 0.05 .

\section{Competing interests}

The authors declare no competing interests.

\section{Authors' contributions}

AP wrote the manuscript and made significant contributions to data analysis. AK prepared the samples for sequencing and made significant contributions to the manuscript. WJF developed the idea for this study and made significant contributions to the manuscript. CK lead the data analysis and made significant contributions to the manuscript. All authors read and approved the final manuscript.

\section{Acknowledgements}

Mosaic trees came to the attention of scientists through the keen observations of the late Mr Kevin Barker of Yeoval NSW. Dr Penny Edwards and Dr Wolf Wanjura of CSIRO kindly showed us the trees and facilitated our work in many ways. We thank the late Mr Herb Healey, the late Mr Kevin Barker, Mr Bruce Lees and Mr Simon Dwyer for allowing us access to their properties. This work was supported by a Discovery grant from the Australian Research Council to WJF (DP0877063).

Received: 21 August 2012 Accepted: 4 January 2013 Published: 20 February 2013

\section{References}

1. Whitham TG, Slobodchikoff CN: Evolution by individuals, plant-herbivore interactions, and mosaics of genetic variability: the adaptive significance of somatic mutations in plants. Oecologia 1981, 49:287-292.

2. Gill DE: Individual plants as genetic mosaics: ecological organisms versus evolutionary individuals. In Plant Ecology. Edited by Crawley MJ. Carlton City: Blackwell Scientific Publications; 1986:321-343.
3. Youssoufian H, Pyeritz RE: Mechanisms and consequences of somatic mosaicism in humans. Nat Rev Genet 2002, 3:748-758.

4. Inagaki $Y$, Hisatomi $Y$, lida S: Somatic mutations caused by excision of the transposable element, Tpn1, from the DFR gene for pigmentation in subepidermal layer of periclinally chimeric flowers of Japanese morning glory and their germinal transmission to their progeny. Theor Appl Genet 1996, 92:499-504.

5. McGregor SE: Insect pollination of cultivated crop plants. Washington DC: USDA; 1976.

6. Gill DE, Chao L, Perkins SL, Wolf JB: Genetic mosaicism in plants and clonal animals. Annu Rev Ecol Syst 1995, 26:423-444.

7. World-Health-Organisation: ARC Section of cancer information - 2008. http:// globocan.iarc.fr/factsheets/populations/factsheet.asp?uno=900." Retrieved 21/08/12.

8. Penfold A, Morrison F: The occurrence of a number of varieties of Euclayptus radiata ( $E$. numerosa) as determined by chemical analyses of the essential oils. Part II. J Roy Soc NSW 1937, 20:375-377.

9. Edwards PB, Wanjura WJ, Brown WV, Dearn JM: Mosaic resistance in plants. Nature 1990, 347:434.

10. Edwards PB, Wanjura WJ, Brown WV: Selective herbivory by Christmas beetles in response to intraspecific variation in Eucalyptus terpenoids. Oecologia 1993, 95:551-557.

11. Lodish H, Berk A, Kaiser C, Krieger M, Scott M, Bretscher A, Ploegh H, Matsudaira P: Molecular cell biology sixth edition. New York: W. H. Freeman and Company; 2008.

12. Eo SH, Doyle JM, Hale MC, Marra NJ, Ruhl JD, DeWoody JA: Comparative transcriptomics and gene expression in larval tiger salamander (Ambystoma tigrinum) gill and lung tissues as revealed by pyrosequencing. Gene 2012, 492:329-338.

13. Cohen D, Bogeat-Triboulot M-B, Tisserant E, Balzergue S, Martin-Magniette M-L, Lelandais G, Ningre N, Renou J-P, Tamby J-P, Le Thiec D, et al: Comparative transcriptomics of drought responses in Populus: a meta-analysis of genome-wide expression profiling in mature leaves and root apices across two genotypes. BMC Genomics 2010, 11:630.

14. Puranik S, Jha S, Srivastava PS, Sreenivasulu N, Prasad M: Comparative transcriptome analysis of contrasting foxtail millet cultivars in response to short-term salinity stress. J Plant Physiol 2011, 168:280-287.

15. Bourgis F, Kilaru A, Cao X, Ngando-Ebongue G-F, Drira N, Ohlrogge JB, Arondel V: Comparative transcriptome and metabolite analysis of oil palm and date palm mesocarp that differ dramatically in carbon partitioning. Proc Natl Acad Sci U S A 2011, 108:12527-12532.

16. Schmid J, Muller-Hagen D, Bekel T, Funk L, Stahl U, Sieber V, Meyer V: Transcriptome sequencing and comparative transcriptome analysis of the scleroglucan producer Sclerotium rolfsii. BMC Genomics 2010, 11:329-345.

17. Padovan A, Keszei A, Wallis I, Foley W: Mosaic eucalypt trees suggest genetic control at a point that influences several metabolic pathways. $J$ Chem Ecol 2012, 38:914-923.

18. Giardine B, Riemer C, Hardison RC, Burhans R, Elnitski L, Shah P, Zhang $Y$, Blankenberg D, Albert I, Taylor J, et al: Galaxy: a platform for interactive large-scale genome analysis. Genome Res 2005, 15:1451-1455.

19. Conesa A, Gotz S, Garcia-Gomez JM, Terol J, Talon M, Robles M: Blast2GO: a universal tool for annotation, visulization and analysis in functional genomics reserach. Bioinformatics 2005, 21:3674-3676.

20. Soltis DE, Smith SA, Cellinese N, Wurdack KJ, Tank DC, Brockington SF, Refulio-Rodriguez NF, Walker JB, Moore MJ, Carlsward BS, et al: Angiosperm phylogeny: 17 genes, 640 taxa. Am J Bot 2011, 98:704-730.

21. Martin D, Aubourg S, Schouwey M, Daviet L, Schalk M, Toub O, Lund S, Bohlmann J: Functional annotation, genome organization and phylogeny of the grapevine (Vitis vinifera) terpene synthase gene family based on genome assembly, FLcDNA cloning, and enzyme assays. BMC Plant Biol 2010, 10:226-248.

22. Külheim C, Yeoh SH, Maintz J, Foley W, Moran G: Comparative SNP diversity among four Eucalyptus species for genes from secondary metabolite biosynthetic pathways. BMC Genomics 2009, 10:452-463.

23. Dudareva N, Pichersky E, Gershenzon J: Biochemistry of plant volatiles. Plant Physiol 2004, 135:1893-1902.

24. Webb H, Külheim C, Rob L, Hamill J, Foley W: The regulation of quantitative variation of foliar terpenes in medicinal tea tree Melaleuca alternifolia. BMC Proceedings 2011, 5:O20.

25. Dudareva N, Andersson S, Orlova I, Gatto N, Reichelt M, Rhodes D, Boland W, Gershenzon J: The nonmevalonate pathway supports both monoterpene and sesquiterpene formation in snapdragon flowers. Plant Biol 2005, 102:933-938. 
26. Borges F, Gomes G, Gardner R, Moreno N, McCormick S, Feijó JA, Becker JD: Comparative transcriptomics of Arabidopsis sperm cells. Plant Physiol 2008, 148:1168-1181.

27. Piper MD, Daran-Lapujade P, Bro C, Regenberg B, Knudsen S, Nielsen J Pronk JT: Reproducibility of oligonucleotide microarray transcriptome analyses. J Biol Chem 2002, 277:37001-37008.

28. Frenkel M, Kulheim C, Jankanpaa H, Skogstrom O, Dall'Osto L, Agren J, Bassi R, Moritz T, Moen J, Jansson S: Improper excess light energy dissipation in Arabidopsis results in a metabolic reprogramming. BMC Plant Biol 2009, 9:12-28.

29. Yang $X, X u$ H, Li W, Li L, Sun J, Li Y, Yan Y, Hu Y: Screening and identification of seed-specific genes using digital differential display tools combined with microarray data from common wheat. BMC Genomics 2011, 12:513-525.

30. Yazaki K: Transporters of secondary metabolites. Curr Opin Plant Biol 2005, 8:301-307.

31. Chen F, Tholl D, Bohlmann J, Pichersky E: The family of terpene synthases in plants: a mid-size family of genes for specialized metabolism that is highly diversified throughout the kingdom. Plant J 2011, 66:212-229.

32. Ossowski S, Schneeberger K, Lucas-Lledó II, Warthmann N, Clark RM, Shaw RG, Weigel D, Lynch M: The rate and molecular spectrum of spontaneous mutations in Arabidopsis thaliana. Science 2010, 327:92-94.

33. Guo M, Rupe MA, Zinselmeier C, Habben J, Bowen BA, Smith OS: Allelic variation of gene expression in maize hybrids. Plant Cell 2004, 16:1707-1716.

34. Serre D, Gurd S, Ge B, Sladek R, Sinnett D, Harmsen E, Bibikova M, Chudin E, Barker DL, Dickinson T, et al: Differential allelic expression in the human genome: a robust approach to identify genetic and epigenetic cis-acting mechanisms regulating gene expression. PLoS Genet 2008, 4:e1000006.

35. Suzuki Y, Hibino T, Kawazu T, Wada T, Kihara T, Koyama H: Extraction of total RNA from leaves of Eucalyptus and other woody and herbaceous plants using sodium isoascorbate. Biotechniques 2003, 34:988-993.

36. Daohong W, Bochu W, Biao L, Chuanren D, Jin Z: Extraction of total RNA from Chrysanthemum containing high levels of phenolic and carbohydrates. Colloids Surfaces B 2004, 36:111-114.

37. Keszei A, Brubaker CL, Carter R, Köllner T, Degenhardt J, Foley WJ: Functional and evolutionary relationships between terpene synthases from Australian Myrtaceae. Phytochemistry 2010, 71:844-852.

38. Ekblom R, Galindo J: Applications of next generation sequencing in molecular ecology of non-model organisms. Heredity 2011, 107:1-15.

doi:10.1186/1471-2229-13-29

Cite this article as: Padovan et al:: Differences in gene expression within a striking phenotypic mosaic Eucalyptus tree that varies in susceptibility to herbivory. BMC Plant Biology 2013 13:29.

\section{Submit your next manuscript to BioMed Central and take full advantage of:}

- Convenient online submission

- Thorough peer review

- No space constraints or color figure charges

- Immediate publication on acceptance

- Inclusion in PubMed, CAS, Scopus and Google Scholar

- Research which is freely available for redistribution 